\title{
Clinical and epidemiologic features of community versus hospital-acquired Clostridium difficile infection
}

\author{
Violeta Molagic ${ }^{1 *}$, Irina Lăpădat ${ }^{1}$, Raluca Mihăilescu', Cristina Popescu ${ }^{1,2}$, Cătălin Tilişcan ${ }^{1,2}$, Raluca Jipa ${ }^{1}$, \\ Mihaela Rădulescu, ${ }^{1,2}$, Daniela Munteanu', Adriana Hristea ${ }^{1,2}$, Ruxandra Moroti ${ }^{1,2}$, Anca-Ruxandra Negru', \\ Iulia Niculescu ${ }^{1,2}$, Roxana Petre', Raluca Năstase ${ }^{1}$, Angelica Tenițăă, Victoria Aramă1,2 \\ From The 10th Edition of the Scientific Days of the National Institute for Infectious Diseases "Prof Dr Matei \\ Bals" \\ Bucharest, Romania. 15-17 October 2014
}

\section{Background}

Clostridium difficile infection (CDI) is an increasingly common hospital-associated infection. There is an increasing awareness in recent years about the impact of community-acquired Clostridium difficile infection (CA-CDI).

\section{Methods}

We enrolled all CDI patients admitted to the Adults III department of the National Institute for Infectious Diseases "Prof. Dr. Matei Balş", Bucharest, between January July 2014. Stool culture, toxin EIA and Cepheid Gene Xpert C. difficile test were used for CDI diagnosis. The subjects were divided into two groups: CA-CDI patients (Group 1) and HA-CDI patients (Group 2). Our objective was to describe the clinical, epidemiologic features and outcome of CA-CDI compared to hospital-associated CDIs (HA-CDI) including the ATLAS bedside severity scoring system. Statistical analyses were performed using SPSS Statistics package v.17.

\section{Results}

We included 57 patients with median age 69 years (IQR $=54 ; 78)$. Male/female ratio was 0.72 . Most patients (75.4\%) presented with an initial CDI episode, the rest having the first (17.5\%) or next (2-5) (7\%) recurrences. The median value of ATLAS score was $3(\mathrm{IQR}=2 ; 4)$. Most patients $(87.7 \%)$ had previously received antibiotic therapy. In $15.8 \%$ cases cancer had been previously diagnosed and

\footnotetext{
* Correspondence: violeta_molagic@yahoo.com

"National Institute for Infectious Diseases "Prof. Dr. Matei Balș", Bucharest, Romania

Full list of author information is available at the end of the article
}

$17.5 \%$ of patients had had recent surgery. Clostridium difficile 027 strain was identified in almost all patients.

The patients were treated with vancomycin $(73.7 \%)$, metronidazole (12.3\%), vancomycin/metronidazole association (10.3\%); 3.5\% received tigecycline. Nine patients $(15.8 \%)$ were included in the CA-CDI and forty-eight patients $(84.2 \%)$ had HA-CDI. The number of CA-CDI in the first 7 months of 2014 was about two times higher than in 2013. Group 1 had fewer comorbidities, were younger (median 52 years (IQR 34.5;77) vs. 69.50 years (IQR 55.25;78), $\mathrm{p}=0.164$, had more mild CDI episode $(55.6 \%$ vs. $33.3 \%, \mathrm{p}=0.26)$, all had received antibiotics and two cases received proton pomp inhibitors.

Group 1 received more aminopenicillins (33.3\%) and less CEPH (11.1\%) compared to Group 2 (2.1\% and $18.8 \%$, respectively, $\mathrm{p}=0.06$ and $\mathrm{p}=1$ ). FQ use was similar: $22.2 \%$ in Group 1 vs. $18.8 \%$ in Group 2. There was one death in HA-CDI. There were no statistical differences between the two groups regarding: sex distribution, median ATLAS score - 3, rates of complicated/recurrent $\mathrm{CDI}$ and use of vancomycin or metronidazole treatment.

\section{Conclusion}

Approximately one sixth of CDIs were CA-CDI. These patients were younger, had predominant mild CDI and received more frequently aminopenicillins than those with HA-CDI. We found no significant differences between the two groups regarding Clostridium difficile 027 strain prevalence and infection severity. 


\section{Authors' details}

'National Institute for Infectious Diseases "Prof. Dr. Matei Balş", Bucharest, Romania. ${ }^{2}$ Carol Davila University of Medicine and Pharmacy, Bucharest, Romania.

Published: 15 October 2014

doi:10.1186/1471-2334-14-57-025

Cite this article as: Molagic et al:: Clinical and epidemiologic features of community versus hospital-acquired Clostridium difficile infection. $B M C$ Infectious Diseases 2014 14(Suppl 7):025.

Submit your next manuscript to BioMed Central and take full advantage of:

- Convenient online submission

- Thorough peer review

- No space constraints or color figure charges

- Immediate publication on acceptance

- Inclusion in PubMed, CAS, Scopus and Google Scholar

- Research which is freely available for redistribution

Submit your manuscript at www.biomedcentral.com/submit
Ciomed Central 